\title{
Quantum States in Templated Biological Processes
}

\author{
Robert Englman \\ Ariel University, Ariel, Israel \\ Email: englmanrobert@gmail.com
}

How to cite this paper: Englman, R. (2021) Quantum States in Templated Biological Processes. Open Journal of Biophysics, 11, 233-251.

https://doi.org/10.4236/ojbiphy.2021.113009

Received: June 3, 2021

Accepted: July 9, 2021

Published: July 12, 2021

Copyright $\odot 2021$ by author(s) and Scientific Research Publishing Inc. This work is licensed under the Creative Commons Attribution International License (CC BY 4.0).

http://creativecommons.org/licenses/by/4.0/ (c) (i) Open Access

\begin{abstract}
Living matter is characterized by its variegated potential energy landscape possessing a proneness to continually absorb externally supplied energy. This enables it to ascend from its momentary energy minimum state to one of its myriad barriers only to subsequently descend to a new minimum with a potentiality to perform new functions or processes, in the while exuding energy (mainly in the form of heat). As in studies of molecular intersystem crossing, the jumping processes are describable in terms of quantum states. In this work we derive the low energy quantum states for those three templated self-assembling processes, self-replication, metabolism and self-repair that are commonly regarded as distinguishing animate from inanimate substance. The outcome of each process is a new, long-living, stable molecular aggregate characterized by its specific conformation, comprising a host of micro-states associated with sub-conformations and patterned upon the template. The provenance of these newly-formed states is obtained here by a unified formalism for all three processes, based on a Hamiltonian, constructed in an abstract Hilbert-space framework, whose essences are bilinear coupling terms in the Hamiltonian between the template and the bath, as well as between the reactants and the bath. Treating these terms by second order perturbation, one finds in low lying quantum states an alignment between the template and the product, somewhat analogous to the Kramers-Anderson superexchange mechanism, with the bath replacing the bridging anion and by exploitation of the decohering due to the randomness of the bath. The idea underlying this work, recurrent in the biological literature and here expressed in a Physics, Hamiltonian framework, is the correlative unity of the whole biological system comprising multiple organs.
\end{abstract}

\section{Keywords}

Living Systems, Self-Organization, Hilbert Space, Molecular Conformation, Decoherence, Pseudo Jahn-Teller-Effect 


\section{Introduction}

The dichotomy of living versus inanimate matter is one of the central puzzles in our understanding of the world around us and as such has occasioned a wide literature [1]-[7]. Besides constituting an intellectual challenge (reflecting also on ideologies [8], beliefs [9], life's meaningfulness [10]), it has bearings on very practical issues, like robotics [11], biomimetics [12], artificial intelligence [13] [14], quantum computing [15] [16]. It is commonly asserted that living systems are distinguished by their faculty for self-replication, metabolism and self-repair (the first of which is accredited to Kant). In this work each faculty is formulated in a quantum mechanical framework. The limitations of such undertaking are so obviously manifold that they need not be stressed at the outset, but will be noted as we go along. The basic rationale is the recognition that biological systems are definite molecular aggregates (in Schrödinger's terminology "aperiodic crystals" [1]) with well defined conformations. The meaning of conformation is the mode of mutual attachment between molecules in an organ, including their relative orientations, but here we include also electronic and vibrational states. In short, all degrees of freedom (dof).

In actual biological systems conformations possess a large measure of stability or permanence, measured erstwhile by the free energy excess of the next, higher lying conformation. Thus in functional proteins this excess in the unfolded state relative to the native, folded state is about $2500 \mathrm{~K}$ in temperature units, so that at room temperatures at most only a fraction 0.00025 of proteins is unfolded [17]. The high conformational stability of DNA is at the root of anthropology analyses of human and animal skeletal remains, lifeless over several millennia (a presumed 700, in the case of a horse in Yukon permafrost in Alaska). The stability of conformations implies that they are residing in a low lying quantum state. Below it is argued that this is a mixed state, composed of micro-states identified as those arising from numerous multi-stable sub-conformations [18] [19] [20]. Under assumption of ergodicity, this entails a decohered motion of a particular organ between its micro-states. The status of states in sub-conformations ("conformational substates", CS) belonging to the same conformation (e.g., to the folded form of proteins) and crucial for their signalling and functions, will be discussed in subsection 3.2, below.

In a unified formalism for each of the three above mentioned conformational changes of biological organs, the present work shows how each CS in the outcome organ is parented by the analogous CS in the template. This is achieved by deriving low-lying eigenstates for the template-product organ pair from a postulated Hamiltonian, whose notable features are the organs' bilinear interaction terms with the environment (the "bath"). Treated perturbationally, these terms align corresponding states in the Hilbert spaces of the pair.

It is stressed that the theory here outlined does not address the mode of functioning of the biological units, an activity that takes place under conditions of non-equilibrium, but only their mode of assembly onto a quiescent form out of 
their constituents. The theory has thus some methodological similarities to what is practised in CASP (Critical Assessment of protein Structure Prediction), in which the task is to predict three dimensional structures of proteins on the basis of submitted various amino acid sequences [21]. Earlier successes in CASP competitions, mainly through the intense computational efforts of Computational Molecular Physics (CMP), have been summarized in [22] and a more recent one described in [23]. Full and partial applications of quantum mechanical methods to biological systems, pioneered in [24], have been comprehensively reviewed in [25]. Nevertheless, the complexity of the molecular arrangements is frequently countered by adoption of a classical or semi-classical approach.

Adjacent to the present theory of creation of organs from a template, is the subject of morphogenesis, addressing the emergence of form in living systems. In a monumental work, structure and patterns in plants and animals were studied from a "physico-mathematical” viewpoint by D'Arcy W. Thomson [26], in which a quotation epitomises also the present work [27]. Deviations from symmetrical forms, due to random disturbances and fortified by activators ("evocators") and inhibitors, were treated with methods of Physical Chemistry by Turing [28]. Recent ramifications of morphogenesis, particularly in the direction of embryogenesis, incorporate a host of assistant actors [29]. A detailed sequential description of the latter is provided in [30].

The following introductory remarks refer mainly to the first of three processes treated here, but are valid for the other two, as well.

In a self-replicative process the template $(\mathrm{T})$ unit (composed of possibly one, but mostly several interwoven molecules) gathers up from the surrounding molecular Zoo, in biological literature named "library", exactly the same molecules of which itself is composed and arranges them with the intermediary of enzymes in a new unit, the replicate (R), that assumes the same (or very nearly so) state as itself and (in case of contact or close adjacency) detaches itself from it to continue with separate existences. (Cf. childbirth as an example or an analogy).

From the Physics viewpoint, in the past self-replication (SR) has been treated as a process, subject to laws of non-equilibrium Thermodynamics or Dynamic Statistical Mechanics (e.g., [31] for a recent account); here it is treated by consideration of the microscopic entities of which it is composed. As such, these are subject, like all other material entities, to Quantum Mechanics (QM), and as a consequence require description in terms of the basic brick-stones of QM, namely "quantal states". It is upon these that the followings rest. SR being a process, implies by definition "transitions" and more precisely in QM: transitions between the states pre- and post-SR; their description is avoided here due to the fact that the status of transitions in QM (termed as "Measurements") is problematic and controversial.

\subsection{Sub-Conformal Protein Motion: Phased or Decohered?}

For proteins (and not for them alone) it is now well established that a profile of 
the potential energy landscape along an interaction coordinate consists of a great number of potential wells of similar depths (energy-wise differing by less than room temperature and separated by barriers of energy heights exceeding $800 \mathrm{~K}$ ) [32] [33]. In their quiescent mode, proteins responding to temperature caused fluctuations move about these wells and the important question arises whether the quantum state of a mobile protein is phase observing, such that in the superposition (sum) of the components belonging to the different wells there persist definite phase relations, or is it a "mixed state" in which the weights of components are fixed statistically (possibly by a Gibbs distribution function for a macro-canonical ensemble ), but not the phases. In the latter case, assuming ergodic behaviour in the protein, the time spent by the molecule in each well is also by the same distribution.

The issue of tunneling versus barrier-jumping has been at the basis of the anomalous temperature dependence of the specific heats at low temperatures of some glasses, whose state has formed a prototype for that in proteins [32]. This was clarified in [34] [35] by considerations of tunneling in two level systems, which is of importance at low temperatures and there alone [34] [35] [36] [37]. Tunneling in biological molecules was the subject of Colloquium in the late seventies [38], in which the question of phase-conserving versus decohered state outcome of the processes was left unanswered.

In the former, passage between the wells may be promoted through temperature effects raising and lowering adiabatically the wells, thus enabling the protein to tunnel across two or more wells coherently. Movement in the mixed state arises by the more common barrier jumping, Marcus-type thermally activated mechanism. If adjacent wells belong to different electronic states, then the electron-vibrational mechanism (associated with an energy-gap law behaviour and involving a "promoting" coordinate factor [39] [40]) is applicable, otherwise only vibrationally induced transitions operate [41]. In either event, the randomising mechanism inherent in temperature activation ensures that in the state resident in the new well there is no remnant of the starting phase. The conclusion that the stable conformation represents a mixed state with respect to the SC states, with no phase coherence among these, so that the different states within the ensemble can give rise to different $\mathrm{H}$-tunneling probabilities. This is of importance for the present theory, in that each SC state in the template gets individually entangled with a state (or states) in the replicate, rather than as a coherent superposition of SC states.

It is of interest to remark that Bersuker has argued in various publications, e.g., [42], that when a point of instability separates the wells, there must necessarily be different electronic states to which the different wells belong, known as the pseudo Jahn-Teller effect. The outcome of such occurrence is the drastic reduction by several orders of magnitudes of the pre-exponential factor in the rate expression from a frequently assumed value of inverse nano- or femto-second [1]. Typical inter-well transition times in some enzymes were indeed estimated to be as high as milliseconds [20]. 


\subsection{Self-Assembly}

The three transformations treated here are those for the normal flow of biological information: DNA can be copied to DNA (DNA replication), DNA information can be copied into mRNA (transcription), and proteins can be synthesized using the information in mRNA as a template (translation), known as the central dogma of biology [43] and predicated at each stage upon the existence of a template. A related subject not considered here is the non-templated self-assembly of (frequently large and biologically significant) molecules [44] [45], in which the final product is not replicated. Successes in this field have strong repercussions in the search for primordial bio-genetic mechanisms [46] [47] [48] [49].

\section{Formalism}

\section{Notation}

Let the full biological system under consideration be considered as composed of a mutually exclusive set of organs, designated generically $X$, each organ possessing an arbitrary number of molecules, describable by $N X_{B}$ bosonic and $N X_{F}$ fermionic degrees of freedom (dof). Although the numbers of quantum states (energy eigen-states, deemed non-degenerate) of these dof are in general infinite (enumerably such for bound states and otherwise for continuous states or for translational degrees of motion), we consider only a finite though arbitrarily large number of these, $M X_{B}$ and $M X_{F}$ respectively, so that the Hilbert space of each organ is of size $N X=N X_{B}^{M X_{B}} * N X_{F}^{M X_{F}}$. The whole Hilbert space is the direct product of these organs. Next, each organ is distinguished by a capital letter with eigenstates labelled with the corresponding greek letter, as follows:

1) The template organ $T$, with state kets $|\tau\rangle$, characterized by stability and being well organized to carry out various tasks.

2) The template's sister organs $S$ (with kets: $|\sigma\rangle$ ) composed of the same types of molecules and in the same configuration and state as $\mathrm{T}$. These organs number $N_{S}$.

3) The candidate for replication $\mathrm{R}(|\rho\rangle)$, also consisting of the same types as the template, but initially in different states, e.g. dispersed in the system.

4) A different set of molecules from the former, out of which some new, product organ is formed, designated $\mathrm{P}(|\pi\rangle)$.

5) The full biological system excluding those items in the above, constituting the bath $\mathrm{B}(|\beta\rangle)$, characterized by the random amplitudes and phases in the states of its constituents.

We now order the points in Hilbert state of each organ according to rising energy values (supposed to be non-degenerate), with the energy ground state labelled 0 . We thus obtain a vector for each organ and the direct product of the five vectors for the whole system. We write this

$$
|\Psi\rangle=|T\rangle|S\rangle|R\rangle|P\rangle|B\rangle
$$

A partial direct product, exclusive of $|X\rangle$ will be denoted $|\Psi\rangle_{X}$ and that 
exclusive of $|X\rangle|Y\rangle,|\Psi\rangle_{X Y}$.

Members of mammoth direct product in Equation (1), numbering

$\Pi_{X=T, S, R, P, B}(N X)$, will form the bases for the Hamiltonian matrix to be formulated. The Hamiltonian operator is a sum of the self-energies of the organs and of coupling terms between the first four organism types and the bath. The latter terms will be treated by perturbation theory, but their entering the formalism is the foundation stone of the theory. Conceptually, they reflect the unity and cohesion of the full living system. We thus start with the Hamiltonian operator

$$
\begin{gathered}
\hat{H}=\sum_{X=T, S, R, P, B} \widehat{H_{X}}+\sum_{X=T, S, R, P} \widehat{H_{X B}} \\
\widehat{H_{X}}=|\Psi\rangle_{X} \sum_{\xi} h_{\xi}|\xi\rangle\left\langle\left.\xi\right|_{X}\langle\Psi|,(X=T, R, S, P, B)\right. \\
\widehat{H_{X B}}=|\Psi\rangle_{X B} \sum_{\xi \beta} g_{\xi \beta}|\xi\rangle\left\langle\left.\beta\right|_{X B}\langle\Psi|+\text { c.C., }(X=T, R, S, P)\right.
\end{gathered}
$$

The operators in the second line enter the Hamiltonian matrix as diagonal terms; those in the third line as off-diagonal terms.

(Remark on the formalism: A more natural description of the systems would be within a second quantization formalism. But then fermionic and bosonic operators would have to be distinguished, thus doubling the nomenclature. An added remark is that in a previous, much more simplified, formulation of the theory [50] we included a further many component system, entitled the "library" which was then incorporated into a Hamiltonian of a few $\approx 2^{5}$ order and solved numerically. As pointed out there, their inclusion did not qualitatively affect the outcomes. Here they are included in the bath. One may also add for clarification, that, with the bath being considered homogeneous and of limitless extent, one may eliminate from the formalism the space-translational dof, keeping only those for the inter-molecular distances.)

With this we are ready to formulate the three salient biological activities: replication, metabolism and repair, in turn.

\section{Self-Replication}

Here one considers two different realizations of the T-R system the template (T) and the (potential) replicate (R), each containing the same molecular set and therefore the same set of dof. We thus obtain two state vectors (kets) of size $N$ each, designated

$$
|T\rangle,|R\rangle
$$

with representatives $T_{\tau}$ and $R_{\rho}$, respectively.

$$
T_{\tau}=\mathrm{e}^{\mathrm{i} \phi_{\tau}} \delta_{\tau, \tau_{g r}} ; \tau_{g r}:=0 ; R_{\rho}=\frac{1}{\sqrt{R_{\text {mix }}}} \mathrm{e}^{i \phi_{\rho}} \delta_{\rho, \rho_{R_{i}}},\left(R_{i}=1, \cdots, R_{\text {mix }}\right)
$$

In the template $T_{g r}$ is an eigenstate, being one or another CS of the conformational energy ground state, e.g., of a folded state for a protein. The (potential) replicate is in one of its $R_{\text {mix }}$ 's state, being a mixed state with equal likelihood of being in any of these. Though the concise notation does not reveal any great dif- 
ference between the states of $\mathrm{T}$ and $\mathrm{R}$, the construction of the state vectors from all degrees of motion allows us to represent the template state by a localized compact and ordered arrangement of molecules and the (potential) replicate state by a set of isomerically different and even dispersed, irregularly spaced and oriented molecular aggregate. The "miracle" is that the formalism shows the way to arrange the latter into the likeness of the orderly agglomerate of the template.

Additional to their being in different eigen-energy states, the difference between $\mathrm{T}$ and $\mathrm{R}$ is also reflected in their initial entropy values being 0 (for $\mathrm{T}$ ) and $\ln R_{\text {mix }}$ (for $\mathrm{R}$ ). The entropy difference is due to the environment (the "bath") being regarded homogeneous and of unlimited extent, in which the freely floating molecules can take up any position, while in the attached situation, they lack this freedom. The gain in free energy resulting from a decrease of entropy at room temperature, incident upon attachment, relative to a free movement for particles at millimolar concentration has been estimated as $5.5 \mathrm{kcal} / \mathrm{mol}$, while for the freezing of rotational freedom the free energy gain was $0.7 \mathrm{kca} / \mathrm{mol}$ [45].

\subsection{Single Subconformation}

\subsubsection{A "Superexchange" Analogy}

Let us consider for a start the case when there is a single well in the conformation; the realistic multi-stability case is taken up in subsection 3.2. For the template to have the capability of self-replication, it must have the property of stability. This means that

1) $\mathrm{T}$ is in ground state $\tau=0$, and to avoid excitation by the rest of the organism or by thermal fluctuation,

2) its energy relative to the next excited state must be negatively much larger than the scaled temperature: $\left|h_{0}\right| \gg k_{B} T,\left|h_{1}-h_{0}\right| \gg k_{B} T$.

( $k_{B}$ being the Boltzmann constant and in this context $T$ the temperature. Henceforth, $k_{B} T$ will serve as the energy unit.)

It needs to be remarked that for identically labelled components of $\mathrm{T}$ and $\mathrm{R}$, $\tau=\rho$, one has $g_{\tau \beta}=g_{\rho \beta}$, since both coefficients couple identical states to a given bath state. With this property and having regard to the random phases of the bath state amplitude we can show, incurring some approximation procedures. that the T-bath and R-bath coupling terms in Equation (4), taken together, force the replicate to a state identical to that of the template. To demonstrate this, we treat the following, arising from two terms in Equation (4), perturbationally.

$$
\widehat{H_{T B}}+\widehat{H_{R B}}:=\widehat{H_{T R-B}}
$$

The first order perturbational correction to the energy due to these terms

$$
\delta^{(1)} E_{\Psi}:={ }_{T R}\left\langle\Psi\left|\widehat{H_{T R-B}}\right| \Psi\right\rangle_{T R}
$$

vanishes due to the randomness in the bath variables. The second order correction is now manipulated as follows:

$$
\delta^{(2)} E_{\Psi}:=\frac{\sum_{\text {exc }}\left|\left\langle\Psi\left|\widehat{H_{T R-B}}\right| \Psi\right\rangle\right|^{2}}{E_{\Psi}-E_{\text {exc }}}
$$


Approximate now by replacing the denominators by their (negative) average $-|\nabla E|$ and apply closure to get

$$
\delta^{2} E_{\Psi} \approx-|\nabla E|^{-1}\left\langle\Psi\left|\left[\widehat{H_{T B}}+\widehat{H_{R B}}\right]^{2}\right| \Psi\right\rangle
$$

Opening the square bracket, one finds that the squared terms contribute only negative constant terms to the T-R system. However, the cross terms give after tracing over the $\mathrm{P}, \mathrm{S}, \mathrm{B}$ organs' dof

$$
-\frac{1}{|\nabla E|}\left\langleT \left|\left\langleR \left|\left[\sum_{\tau \rho \beta} g_{\tau \beta}^{*} g_{\rho \beta}|\tau\rangle|\rho\rangle\langle\rho|\langle\tau|] \mid R\rangle|T\rangle+\right.\right.\right.\right.\right.\text { complex conjugate }
$$

whereas for $\tau \neq \rho$ the sum decoheres due to the random nature of the bath, for $\tau=\rho$ in the sum the product terms $g_{\tau \beta}^{*} g_{\rho \beta}$ are necessarily positive $\left|g_{\rho \beta}\right|^{2}$, since both the template and the replicate are composed of the same set of molecules and the interaction with the bath is identical. Adopting now from the theory of electron-paramagnetic resonance a further approximative procedure, known as the "Effective Hamiltonian formalism", we can employ the term inside the bra-kets as an interaction term in the T-R Hamiltonian. Explicitly

$$
\delta H_{T-R}=-\sum_{\tau \rho}|\tau\rangle|\rho\rangle W_{\tau}\langle\rho|\left\langle\left.\tau\left|; W_{\tau}:=-\frac{1}{|\nabla E|} \sum_{\beta}\right| g_{\tau \beta}\right|^{2}\right.
$$

The direct effect of this term is the tendency to align in the ground state the replica state with the template state. As by construction and its stability (expressed by $h_{\tau=0} \gg 1$ for the template's state), the replicate's state will also be this state, provided only that algebraically

$$
W>h_{\rho} \text {, all } \rho
$$

With the inclusion of the term gained from the second order perturbation and after elimination of the bath' dof, the Hamiltonian for the T-R system reads

$$
\begin{aligned}
\widehat{H_{T-R}} \approx & -\sum_{\tau \rho, \tau^{\prime} \rho^{\prime}} h_{\tau}|\tau, \rho\rangle\left\langle\tau^{\prime}, \rho^{\prime}\left|\delta_{\tau, \tau^{\prime}}-\sum_{\tau \rho, \tau^{\prime} \rho^{\prime}} h_{\rho}\right| \tau, \rho\right\rangle\left\langle\tau^{\prime}, \rho^{\prime}\right| \delta_{\rho, \rho^{\prime}} \\
& +\sum_{\tau \rho, \tau^{\prime} \rho^{\prime}}\left[g_{\tau \rho, \tau^{\prime} \rho^{\prime}}-W_{\tau} \delta_{\tau, \rho} \delta_{\tau^{\prime}, \rho^{\prime}}\right]|\tau, \rho\rangle\left\langle\tau^{\prime}, \rho^{\prime}\right|
\end{aligned}
$$

The first requirement upon this expression is the large value of $\left|h_{0}\right| \gg 1$ in the first sum, thereby ensuring that in the lowest lying energy state of the (diagonalized) $H_{T-R}$, the target ground state $\tau=0$ will be predominant. Next is the requirement for a large diagonal term $W_{0} \gg 1$ in the square brackets of the second line, that has the role of making the

$$
\rho=\tau=0
$$

state govern also the replicate in the T-R ground state, and this under the proviso that in the square bracket the accompanying term $g_{\tau \rho, \tau^{\prime} \rho^{\prime}}$ is substantially less. The criteria for SR can thus be quantitatively formulated through the inequalities on a temperature energy scale $\left(k_{B} T=1\right)$

$$
h_{\tau} \gg 1 \text { for } \tau=0,\left|h_{\rho}\right|=O(1) \text { all } \rho, W_{0} \gg 1, W_{0}>\left|g_{\tau \rho, \tau^{\prime} \rho^{\prime}}\right| \text { all indices }
$$

Here the second relation ensures that self replication occurs as an outcome of 
the process of switching on the interactions and not due to the intrinsic property of the replicate. The physical meanings of the relations are for the first: the stability of the template; for the second: a propensity of change in the initial state of the (potential) replicate; for the third: an effective interaction with the bath; finally, the low availability of alternative channels for changes in the replicate.

The similarity of the formalism with Kramers' and P.W. Anderson's mechanism for superexchange [51] [52] will be noted, with the bath replacing the bridging anion and exploitation of the randomness of the former. The aligning factor $W_{\tau}$, Equation (12), has its counterpart in the "attractive coupling" expression (based on third order perturbation) of Anderson following his Equation (19) in [52].

\subsubsection{An Illustration}

The aligning effect of the second order perturbation term can be seen in an algebraically solvable model, in which the template and the replicate have each two states. With the choice of a large value for the template's self-energy $h_{T}=24$, the calculated alignment of the replicate in the ground state is contour plotted in Figure 1, as function of $W$ as the abscissa and $h_{R}$ as the ordinate. In the blank bottom-right region the alignment is complete, while in the blue upper-left part the alignment is well-nigh zero. The diagonal dividing line is $\left|h_{\rho}\right|=W$, a line of bifurcation, along which the aligning and non-aligning states are co-degenerate. (A more detailed study of this bifurcation, potentially giving rise to criticality, is left to a future work.)

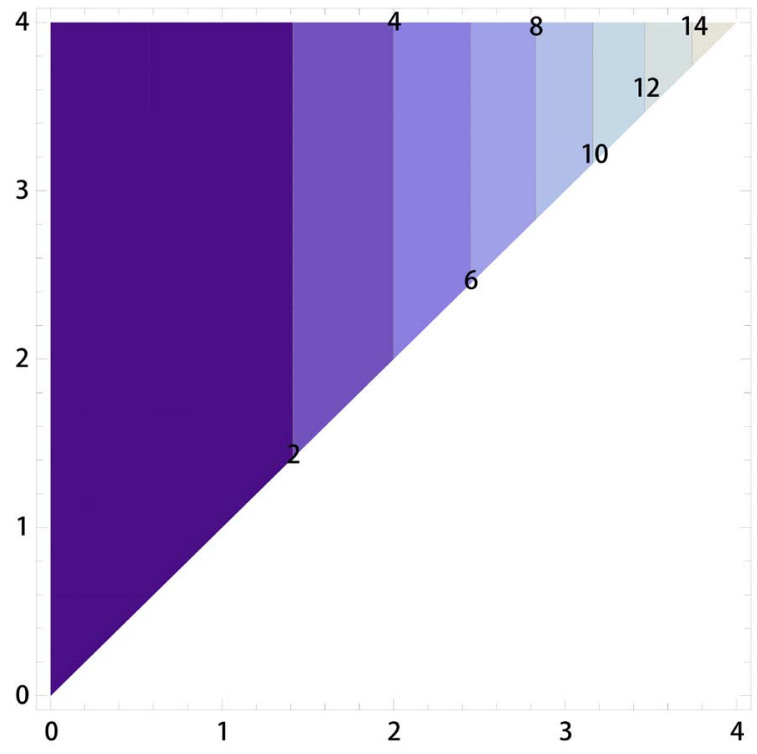

Figure 1. Alignment of the replicate with the state of the template plotted with $x=W$ (the second order bath coupling strength); $y=-h_{\rho}$ (a negative, anti-aligning replicate self-energy). There is full alignment in the blank triangle, minimal alignment in the blue regions, with more smallness on the left. The gradations show changes in the minute alignment probabilities for the non-aligned region roughly in units of $3 \times 10^{-4}$ decreasing towards the left. 


\subsection{Multiple Subconformations, SC}

While the foregoing theory related to a single ground state SC for both the template and the replicate, in reality there are a number of these, designated subconformations (SC), belonging to the same generically termed "conformation", e.g., the folded state for proteins. Moreover, these SC are vital for the functionings of the organ, insomuch that an external signal, as also thermal fluctuations shunt the organs between the SC, while still residing in the basic conformation. As the energy difference between SC are much smaller than between different conformations, they will be populated to varying extents and the interconversions are governed by the heights of the barriers between the subconformations [18]. Likewise, the activity of the protein, like H-tunneling, may send it from one to another SC. More radical and lasting effects were noted in [19] under the heading of "evolvability", with this taking place at a faster rate in RNA than in proteins.

An examination of the foregoing theory shows that the results of Section 3 hold for each SC separately, in the sense that when the template resides in one of its multi-stable states, it will drag along the replicate to an identical multi-stable state. The protein being in a mixed state of SC, there is no phase relation between them. The process is illustrated in Figure 2. Formally, one extends the notation of 0 for a unique ground state to $0^{\prime}, 0^{\prime \prime}, 0^{\prime \prime \prime}, \cdots$ for the set of multi-stable states, and replace the criterion in Equation (15) to

$$
g_{\rho \beta}=g_{\tau \beta}, \rho=\tau=0^{\prime}, 0^{\prime \prime}, 0^{\prime \prime \prime}, \cdots
$$

\section{Metabolism}

Here the situation differs from the one for SR, in that the physical organ performing the metabolic action (which due to the scarcity of symbols will still be termed "template, T" and its Hilbert space labelled by $\tau$, in spite of the risk of misnomers) gathers up from the "library" a set of molecules different from those that itself is composed of, organizes, transforms (metabolises) them to a new aggregate of a specific form and potentiality to perform a specific task and-then

(a)

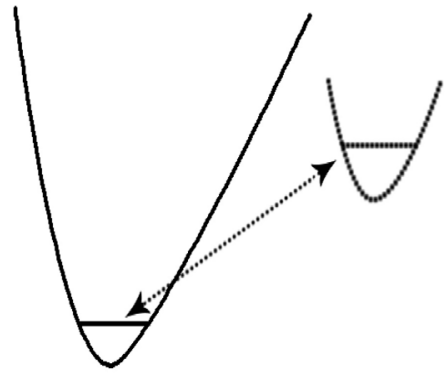

(b)

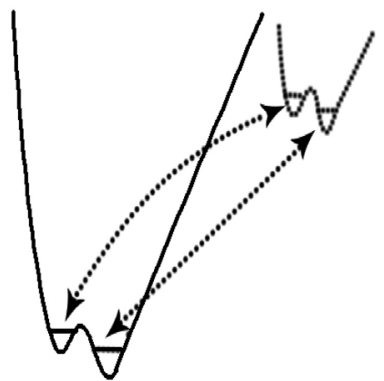

Figure 2. Replication to a CS. (a) For a single CS in the conformation, the unique ground state in the template (lower well) is replicated onto the replicate (upper well) to form the combined ground state; (b) For multiple CS (with only two drawn) each CS in the template is separately partnered by the corresponding CS in the replicate. 
sends the away. In the present formalism the product is labelled $\mathrm{P}$ and the components $\pi$. The most notable examples are for $\mathrm{T}=\mathrm{DNA}$ and $\mathrm{P}=$ a protein.

The required changes in the formalism set out for SR are, apart from the obvious replacements of $R \mapsto P, \rho \mapsto \pi$, that the Hilbert-spaces of $\mathrm{T}$ and $\mathrm{P}$ are different. The main step now is the inclusion of the perturbative terms

$$
\widehat{\nabla H_{T B}}+\widehat{\nabla H_{P B}}:=\widehat{H_{T P-B}}
$$

in the Hamiltonian operator. When the effects of these are estimated by second order perturbation theory and following the approximative procedures (constant, average energy denominators, random phase dissipation, effective Hamiltonian description) made in Section 3, one obtains the following equalities that are necessary and sufficient for the creation of a new metabolic entity, fully specified, apart from phase, by the Hilbert space index $\pi_{P}$ :

$$
g_{\tau_{g r}, \beta}=g_{\pi_{p}, \beta} \text {, all } \beta \text {, the bath-state index }
$$

This relation ensures that all bilinear terms in the effective T-P Hamiltonian term add up constructively and negatively. Analogously to the result for SR, when this term has a large absolute value, one obtains for the T-P ground state the new combined state made up of the original template state $\tau_{g r}$, as well as of the new product state $\pi_{P}$, a metabolic addition to the organs of the biological system. The identity and form of this product state are specified by that, that it is the state $\left|\pi_{P}\right\rangle$ for which the equalities in Equation (19) hold. (By implication, if these equalities do not hold, the template does not metabolize and is left to do other tasks.)

At this point it needs to be noted that (in contrast to the case of SR, in which the two members of Equation (19) related to the states of identical molecular systems, in the template and its replicate, and had clear intuitive justification) the provenance of Equation (19) is not clear. It may be related to some underlying unity in the full biological system, T-P-B, possibly beyond the reach of human inquiry. Nevertheless, the foregoing analysis is of value as a comprehensive descriptive formalism within a quantum mechanics frame.

\section{Biological Repair}

Here matters are more complicated, since there are three stages to the process: the recognition of damage, (frequently) sealing and healing (regeneration) [12]. For each stage questions arise as to how the information reaches the organ, where it originates, what prompts the need and mode of action to be taken, etc.? These questions are not here answered. There exists, though, for the threshold timing of wound recognition in cells an estimate of $1-10 \mathrm{~s}$, provided in [53], which discusses in detail two instances of damage: a cell damage due to puncture of Xenopus oocytes and the loss of oral apparatus in Stentor coeruleus.

The latter form is clearly the more radical one, exemplifying that class of damage in which a loss of an entire organ has taken place (the loss of flagellum in Chlamydomonas is a frequently quoted example, as again in [53]); even here, 
too, some repair or adjustment is frequently brought into play. In addition to repair, or as an independent side effect, a genetic regulatory mechanism provoked by organ changes has also been noted in some cases [54]. Thus in observations in amphibian embryos of polyploidy (the presence of more than two chromosome sets, frequent among plants) changes in cell shapes were noted, thereby maintaining the normal form of organs. Remarkably, the theory presented in the following subsumes even this radical class of damages, the loss of an organ, though it does so in an extremely abstract way. A criterion for catastrophic, irreparable damage types also emerges from the formalism.

\subsection{A Majority Rule}

The guiding idea for recognition of aberrations by biological organs and their subsequent elimination may be termed "the democratization of the organs". It postulates that any time of maturation there are a number of organs (cells, proteins, etc.), entrusted to carry out identical tasks, and at stages of normalcy possessing the same structure and occupying the same quantum state. Normally, this is their ground state (0). However, it can happen that some external factor removes temporarily one or a few organs from that state forcing it into another damaged state, but leaving the majority of organs in their original ground state. At this stage, there enters the interaction between the organs, by which the sister organs notify the damaged state of its aberrant status. At that instance, the healing or repair process begins.

This arises from the mutual interaction between the damaged organ and its sister organs, mediated by the coupling of the organs to the bath. As in the previous Sections 3 and 4 this coupling is treated in second order and with tracing over the bath states. Due to its entering negatively the Hamiltonian, the resulting coupling drives the damaged organ to an alignment with the healthy sister organs' state, namely to its pre-damage ground state. Needless to re-emphasize that the theory (which is formulated in terms of states, not of processes) does not explain the mechanism of the events (recognition, repair), only their outcomes. The following separates the stages of the repair process.

\subsubsection{Damage}

We recall from Section 2 that the stability or permanency of the template $\mathrm{T}$ is ensured by that its self-energy $h_{0}$ in its ground state (labelling $\tau=0$ ) is negative and numerically large. We now adopt the symbol $D$ for the organ undergoing damage and $\delta$ for its states (the ordered Hilbert-space points), so that again $\delta=0$ denotes the ground state of $\mathrm{D}$ prior to its repair. As parts of the living system, additional to T there are $N_{S}$ sister organs labelled $n$ tasked with the same function as $\mathrm{T}$, identical to it, all residing in their ground states.

The damage done to $\mathrm{T}$ is expressed by the application of a unitary, transformation matrix $D_{\delta, \tau}$ to the template T. During its damaged state the organ's states are

$$
|\delta\rangle=\sum_{\tau} D_{\delta, \tau}|\tau\rangle
$$


with self-energies

$$
E_{\delta}=\sum_{\tau}\left|D_{\delta, \tau}\right|^{2} h_{\tau}
$$

Clearly the ground state energy of this, $E_{g r} \approx-\left|h_{0}\right|\left(D_{g r, 0}\right)^{2}$ is different from that of the undamaged template $-\left|h_{0}\right|$. Moreover, the new damaged ground state of the organ is $\sum_{\tau} D_{g r, \tau}|\tau\rangle$, different from $|0\rangle$. (The difference in the state, arising from the damage can be of any form or extent; e.g., it can even represent the detachment of part of the organ. This follows from the completeness of the Hilbert space, which includes all possible states and configurations of the ensemble of molecules constituting the organ.)

\subsubsection{Repair}

The repair is achieved by the damaged organ receiving feedback from the rest of the sister-organs in the system. As in the previous Sections 3 and 4 the correction arises from the second order perturbational treatment of the interaction terms of the template and its sister molecules with the bath

$$
\widehat{H_{D B}}+\sum_{n=1, \cdots, N_{S}} \widehat{H_{S_{n} B}}:=\widehat{H_{D S-B}}
$$

similar to Equation (7), but a sum over all sister organs in the second term on the left. It is important to realize that the organ-bath coupling terms $g_{\tau \beta}$ in $H_{D B}$ are not changed by the occupation of the states, since the Hamiltonian depends only on the identity of molecular components and not on the temporary occupancy of the states. This identity is not changed by damage. Therefore

$$
\widehat{H_{D B}} \equiv \widehat{H_{T B}}
$$

Carrying out the second order perturbation as heretofore, one is left with a (negative) coupling of the form

$$
\delta H_{D-S}=\delta H_{T-S}=-\sum_{\tau \sigma}|\tau\rangle|\sigma\rangle W_{\tau}^{D}\langle\sigma|\left\langle\left.\tau\left|; W_{\tau}^{D}:=-\frac{N_{S}}{|\nabla E|} \sum_{\tau \beta}\right| g_{\tau \beta}\right|^{2}\right.
$$

Meaning: The combined effect of the healthy sister organs, residing in their ground state $\sigma=0$ drives the damaged organ back to its original ground state $\tau=0$. Repair has been achieved. In fact, this process of self-repair is equivalent to that in self-replication, since the same molecular entities are involved, the difference being only that here a number $\left(N_{S}\right)$ of organs reproduce their own image.

(As a corollary one may note that with a lethal damage, when the number of undamaged organs $N_{S}$ is insufficiently large, repair may not take place and the system becomes defunct. This can also occur when the system is small and cannot provide appropriate feedback.)

\section{Couplings}

In the foregoing formalism an essential and critical role was played by the interaction terms (of the type $g_{\tau \beta}$ ) between the biological entity and its surround- 
ings. Whereas it is usual to deal with these interactions under the amorphous title of "weak thermal interaction", here they were subject to precise, detailed equalities. Moreover, the entities so being coupled were not confined to the immediate neighbourhood, but were rather spread out over the fullness of the biological space. As already noted elsewhere, organisms' (and also cells') functionality and even survival is not an intrinsic property of the organism (or cell), but is due to interactions between a large number of cells [55]. This property is at the basis of the formalism.

A quotation (with permission) from [55] (p.72) amplifies this, as follows:

"A cell is the basic unit of life and all living organisms are composed of one or more cells. The capacity of organisms to adapt to changing and often hostile environments, tolerate limited failures and heal damaged organs is not because of the robustness of individual cells, but because of the interactions between large numbers of cells. A cell is able to divide into two daughter cells, emit chemicals to the surrounding environment, and actively deform by applying physical forces across its walls. Different chemicals within and around the cell control these actions. A cell can sense chemicals on its walls, as well as in its environment. The nucleus of the cell contains DNA, which encodes different genes that have been retained through evolution. A gene is activated when a certain condition is true. This condition could be a critical concentration level of a chemical or a set of chemicals that the cell possesses or that the cell has sensed. When a gene is activated it may cause certain cell actions, which could result in the turning other genes on or off."

However, question arises as to the origin and nature of these couplings?

Electromagnetic, covalent and exchange forces (or in phenomenological terms: Coulomb, hydrogen bonding, van der Waals and hydrophobic interactions) are prevalent in chemical bondings and as long as the distance dependence of the coupling coefficients is not clarified, it is not possible to answer the question above. It is necessary to note, though, that the coupling terms connect up the organ with the bath (manifest through the bath index $\beta$ in the coupling coefficient), and the bath environment is regarded as homogeneous and of unlimited extent, devoid of any distance dependence from the organ. Still, there is one more possibility for the couplings (one, that is novel and speculative, rather than established), namely that they reflect the entanglement between the entities. The quantum mechanical entanglement effect is distance independent, this being the basis of the Einstein-Podolsky-Rosen paradox, and its occurrence is predicated only by the different dof having been in conjunction once upon a time. This demand is undoubtedly satisfied by the zygote stage of embryonic development, from which animal organs eventually emerge.

\section{Conclusions}

It may be objected that an attempt to portray biological entities as microscopic systems is a futile undertaking, due to their complexities, but it should be noted 
that for long decades the measurement problem in QM was treated, by von Neumann and subsequently, in a similarly abstracted form, in spite of most measurement apparatus having complexities approaching those of biological systems [56] [57]. (In a Wigner formulation of the measurement problem even the experimenter's brain (mind ?) was added to good measure, as a determinant of the physical state.) Alike to the von Neumann formulation of the measurement process, the durations of the three processes (SR, metabolism, self repair) are here left out of considerations.

Regarding the interface of the present work's methodology with the very extensive philosophical discussions on causation, in their terminology this methodology is set on a bottom-to-top approach, contrasting with the advocacy of the opposite, top-to-bottom explications, strongly argued in [58]. In this context, the title phrase "More is Different" due to the eminent physicist P.W. Anderson is sometimes cited as a call to arms for phenomenological descriptions and even the superfluousness of research at a more basic level. In fact, a proper reading of Anderson's article shows that his advocacy was for a steadfast funding of phenomenological research, conducted mainly in the sciences of condensed matter and against the mega-funding of particle physics. This was in line with the view, expressed by him at occasions, that greatly significant advances in Physics take place mainly through novel experimental results (achieved frequently thanks to refined techniques), to be followed by a careful reading of the findings by theoreticians.

In the last four decades we have witnessed numerous, perhaps thousands of intensive computational efforts to elucidate the quantum mechanical basis of specific biological structures and activities [25]. The present work is in a different direction: a formal blueprint set out in general terms. It does not reduce the need for further work of the former kind.

\section{Acknowledgements}

Thanks are due to Eli Eisenberg (Tel-Aviv University) and to Judith P. Klinman (Berkeley University CA) for their remarks and to Noam Kayser (Bar-Ilan University) for technical help.

\section{Endnote Added after Acceptance}

The equilibrated-quantum-states, holistic approach proposed in this paper, contrasting with the conventional non-equilibrium description of living matter's functioning, possesses an analogy with theories of electrical conduction which, after generations of being viewed by the Drude theory as a non-equlibrium phenomenon, has been superseded by a scattered quantum-state treatment (through inclusion of the leads as part of the system) in the Büttiker-LandauerImry theory [59].

\section{Data Availability}

The data that support the findings of this study are available from the author 
upon reasonable request.

\section{Conflicts of Interest}

The author declares no conflict of interest.

\section{References}

[1] Schrödinger, E. (1944) What Is Life? The Physical Aspect of Living Cell. University Press, Cambridge.

[2] Bernal, J.D. (1951) The Physical Base of Life. Routledge and Kegan Paul, London.

[3] Prigogine, I. (1975) Dissipative Structures, Dynamics and Entropy. International Journal of Quantum Chemistry, 9, 443-456. https://doi.org/10.1002/qua.560090854

[4] Dyson, F. (1980) Origins of Life. Cambridge University Press, Cambridge.

[5] Ruelle, D. (2017) The Origin of Life Seen from the Point of View of Non-Equilibrium Statistical Mechanics. arXiv: 1701.08388

[6] Mayr, E. (1982) The Growth of Biological Thought. Harvard University Press, Cambridge, MA.

[7] Brooks. R.A. (2001) The Relationship between Matter and Life. Nature, 409, 409-411. https://doi.org/10.1038/35053196

[8] Hull, D.L. and Ruse, M. (2009) The Cambridge Companion to the Philosophy of Biology. Cambridge University Press, New York.

[9] Sarkar, K. (2007) Doubting Darwin? Creationist Designs on Evolution. Blackwell Publishing, Malden.

[10] Sacks, J. (2011) Chapter 10. In: The Great Partnership, God, Science and the Search for Meaning, Hodder and Stoughton, London.

[11] Sun, L., Yu, Y. and Chen, Z. (2020) Biohybrid Robotics with Living Cell Activators. Chemical Society Reviews, 49, 4043-4069. https://doi.org/10.1039/D0CS00120A

[12] Speck, O. and Speck, T. (2019) An Overview of Bioinspired and Biomimetic Self-Repairing Materials. Biomimetics, 4, Article No, 26.

https://doi.org/10.3390/biomimetics4010026

[13] Emmeche, C. (1994) The Garden in the Machine: The Emerging Science of Artificial Life. Princeton University Press, Princeton.

[14] Etxeberria, A. (2002) Artificial Evolution and Life-Like Creativity. Leonardo, 35, 275-281. https://doi.org/10.1162/002409402760105271

[15] Abbott, D., Davies, P.C.W. and Pati, A.K. (Eds.) (2008) Quantum Aspects of Life, Foreword by Sir Roger Penrose. Imperial College Press, London.

[16] Lloyd, S. (2008) Quantum Computing. In: Abbott, D., Davies, P.C.W. and Pati, A.K., Eds., Quantum Aspects of Life, Foreword by Sir Roger Penrose, Imperial College Press, London.

[17] Wilke, C.O. and Bloom, J.D. (2016) Protein Folding and Protein Stability. In: Kliman, R.M., Ed., Encyclopedia of Evolutionary Biology, Elsevier, New York, 339-343. https://doi.org/10.1016/B978-0-12-800049-6.00180-3

[18] Smock, R.G. and Giorash, L.M. (2009) Sending Signals Dynamically. Science, 324, 198-203. https://doi.org/10.1126/science.1169377

[19] Tokuriki, N. and Tawfik, D.S. (2009) Protein Dynamics and Evolvability. Science, 324, 203-207. https://doi.org/10.1126/science.1169375

[20] Daniel, R.M., Dunn, R.V., Finney, J.L. and Smith, J.S. (2003) The Role of Dynamics 
in Enzyme Activity. Annual Review of Biophysics and Biomolecular Structure, 32, 69-92. https://doi.org/10.1146/annurev.biophys.32.110601.142445

[21] Moult, J., Pedersen, J.T., Judson, R. and Fidelis, K. (1995) A Large-Scale Experiment to Assess Protein Structure Prediction Methods. Proteins. Structure, Function, and Bioinformatics, 23, 2-5. https://doi.org/10.1002/prot.340230303

[22] Birni, F., Simmerling, C. and Dill, K. (2020) Protein Storytelling through Physics. Science, 370, Article No. eaaz3041. https://doi.org/10.1126/science.aaz3041

[23] Callaway, E. (2020) 'It Will Change Everything': Deep Mind's AI Makes Quantum Leap in Solving Protein Structures. Nature, 588, 203-204.

https://doi.org/10.1038/d41586-020-03348-4

[24] Warshel, A. and Levitt, M. (1976) Theoretical Studies of Enzymic Reactions: Dielectric, Electrostatic and Steric Stabilization of the Carbonium Ion in the Reaction of Lysozyme. Journal of Molecular Biology, 103, 227-249.

https://doi.org/10.1016/0022-2836(76)90311-9

[25] Brunk, E. and Rothlisberger, U. (2015) Mixed Quantum Mechanical/Molecular Mechanical Molecular Dynamics Simulations of Biological Systems in Ground and Electronically Excited States. Chemical Reviews, 115, 6217-6263.

https://doi.org/10.1021/cr500628b

[26] Thompson, D’A.W. (1992) On Growth and Form. Dover, New York.

[27] Thompson, D.A.W. (1992) But of the Construction and Growth and Working of the Body, as of All Else That Is of the Earth and Earthy, Physical Sciences Is, in My Humble Opinion, Our Only Leader and Guide. In: On Growth and Form, Dover, New York, 13.

[28] Turing, A.M. (1952) The Chemical Basis of Morphogenesis. Philosophical Transactions of the Royal Society B, 237, 37-72.

https://doi.org/10.1038/d41586-020-03348-4

[29] Gordon, N.K. and Gordon, R. (2016) Embryogenesis Explained. World Scientific Publishing Company, Singapore.

[30] Slack, J.M.W. (2013) Essential Developmental Biology. 3rd Edition, John Wiley, New York.

[31] England, J. (2020) Every Life Is on Fire. How Thermodynamics Explains the Origin of Living Things. Basic Books, New York.

[32] Ansari, A., Berendsen, J., Browne, S.F., Frauenfelder, H., Iben, I.E., Sauke, T.B., et al. (1985) Protein States and Proteinquakes. Proceedings of the National Academy of Sciences of the United States of America, 82, 5000-5004.

https://doi.org/10.1073/pnas.82.15.5000

[33] Elber, R. and Karplus, M. (1987) Multiple Conformational States of Proteins: A Molecular Dynamics Analysis of Myoglobin. Science, 235, 318-321. https://doi.org/10.1126/science.3798113

[34] Phillips, W.A. (1972) Tunneling States in Amorphous Solids. Journal of Low Temperature Physics, 7, 351-360. https://doi.org/10.1007/BF00660072

[35] Anderson, P.W., Halperin, B.I. and Varma, C.M. (1972) Anomalous Low Temperature Properties of Glasses and Spin-Glasses. The Philosophical Magazine, 25, 1-9. https://doi.org/10.1080/14786437208229210

[36] Gillan, M.C. (1987) Quantum-Classical Crossover of the Transition Rate in the Damped Double Well. Journal of Physics C: Solid State Physics, 20, 3621-3641. https://doi.org/10.1088/0022-3719/20/24/005

[37] Khomenko, D., Scalliet, C., Berthier, L., Reichman, D.R. and Zamponi, F. (2020) 
Depletion of Two-Level Systems in Ultrastable Computer-Generated Glasses. Physical Review Letters, 124, Article ID: 225901. https://doi.org/10.1103/physrevlett.124.225901

[38] Chance, B., Devault, D.C. and Frauenfelder, H. (1979) Tunneling in Biological Systems. Academic Press, New York.

[39] Fried, K.F. (1972) The Theory of Radiationless Processes in Polyatomic Molecules. Topics in Current Chemistry, 31, 105-139. https://doi.org/10.1007/BFb0051237

[40] Englman, R. and Jortner, J. (1970) The Energy Gap Law for Radiationless Transitions in Large Molecules. Molecular Physics, 18, 145-164.

https://doi.org/10.1080/00268977000100171

[41] Englman, R. (1979) Non-Radiative Decay of Molecules and Ions in Solids. North Holland, Amsterdam.

[42] Bersuker, I.B. (2006) The Jahn-Teller Effect. Cambridge University Press, Cambridge, Section 4.1. https://doi.org/10.1017/CBO9780511524769

[43] Crick, F.H. (1958) On Protein Synthesis. In: Sanders, F.K., Ed., Symposia of the Society for Experimental Biology, Number XII: The Biological Replication of Macromolecules, Cambridge University Press, Cambridge, 138-163.

[44] Rechtsman, M., Stillinger, F. and Toquato, S. (2006) Designed Interaction Potentials via Inverse Methods for Self Assembly. Physical Review E, 73, Article ID: 011406.

https://doi.org/10.1103/PhysRevE.73.011406

[45] Whitesides, G.M., Mathias, J.P. and Seto, C.T. (2019) Molecular Self-Assembly and Nanochemistry: A Chemical Strategy for the Synthesis of Nanostructures. Science, 254, 1312-1319. https://doi.org/10.1126/science.1962191

[46] Zelinski, W.S. and Orgel, L.E. (1987) Autocatalytic Synthesis of a Tetranucleotide Analogue. Nature, 327, 346-347. https://doi.org/10.1038/327346a0

[47] Feng, Q., Park, T.K. and Rebek Jr., J. (1992) Cross-Over Reaction between Synthetic Replication Yield Active and Reactive Recombinants. Science, 256, 1179-1180. https://doi.org/10.1126/science.256.5060.1179

[48] Luther, A., Brandsch, R. and von Kiederowski, G. (1998) Surface Promoted Replication and Exponential Amplification on DNA analogues. Nature, 396, 245-248. https://doi.org/10.1038/24343

[49] Szosztak, J.W. (2012) The Eightfold Path to Non-Enzymatic RNA Replication. Journal of Systems Chemistry, 3, Article No. 2.

https://doi.org/10.1186/1759-2208-3-2

[50] Englman, R. (2021) A Quantum State Scenario for Biological Self-Replication. Open Journal of Biophysics, 11, 159-176. https://doi.org/10.4236/ojbiphy.2021.112005

[51] Kramers, H.A. (1934) L'interaction entre les atomes magnéténes dans un cristal paramagnétique. Physica, 1, 182-192. https://doi.org/10.1016/S0031-8914(34)90023-9

[52] Anderson, P.W. (1950) Antiferromagnetism. Theory of Superexchange Interaction. Physical Review, 79, 350-358. https://doi.org/10.1103/PhysRev.79.350

[53] Tang, S.K.Y. and Marshall, W.F. (2017) How Single Cells Heal Membrane Ruptures and Restore Lost Structures. Science, 356, 1022-1025. https://doi.org/10.1126/science.aam6496

[54] Fankhauser, G. (1945) The Effects of Changes in Chromosome Number on Amphibian Development. Quarterly Review of Biology, 20, 20-78. https://doi.org/10.1086/394703

[55] George, S., Evans, D. and Marchette, S. (2003) A Biological Programming Model for Self-Healing. Proceedings of the 2003 ACM Workshop on Survivable and 
Self-Regenerative Systems. In Association with 10 th ACM Conference on Computer and Communications Security, Fairfax, October 2003, 72-81.

https://doi.org/10.1145/1036921.1036929

[56] von Neumann, J. (1932) Mathematische Grundlagen der Quantenmechanik. English Edition, Verlag Julius Springer, Berlin. (English Edition)

[57] Araki, H. and Yanase, M.M. (1960) Measurement of Quantum Mechanical Operators. Physical Review, 120, 622-626. https://doi.org/10.1103/PhysRev.120.622

[58] Levin, M. (2020) The Biophysics of Regenerative Repair Suggests New Perspectives on Biological Causation. BioEssays, 42, Article No. 1900146.

https://doi.org/10.1002/bies.201900146

[59] Imry, Y. and Landauer, R. (1999) Conductance Viewed as a Transmission. Reviews of Modern Physics, 71, S306-S312. https://doi.org/10.1103/RevModPhys.71.S306 